\title{
Quince años de experiencia clínica con hidatidosis
}

\author{
Alberto Fica, Andrés Soto, Jeannie Slater, Marcial Peralta, \\ Roberto Humeres, Marcelo Castro, Jaime González y Thomas Weitzel
}

\section{Clinical presentation of hydatid disease during 15 years: a case series from Santiago, Chile}

Introduction: Hydatidosis is a neglected parasitic disease that is endemic in Chile. We present the clinical experience of a single center in Santiago from 1996 to 2010. Methods: Cases were identified using hospital's database. Clinical and pathological features, treatment and outcomes were retrospectively analyzed. Results: In total, 23 patients were identified requiring 30 surgical interventions. Median age was 40 years old (range 5 to 73 ), and $76.5 \%$ visited or resided in regions of known endemicity in Chile. Abdominal cysts were predominant (78.3\%), and most patients were symptomatic (73.9\%). Elimination of cyst material by cough was reported in $42.9 \%$ of patients with symptomatic thoracic cysts. Eosinophilia was present in $41.2 \%$ of patients, and $57.1 \%$ had positive serology for hydatidosis. All patients underwent surgical treatment, in $60.9 \%$ in combination with albendazole treatment. While the majority of liver cysts $(88.9 \%)$ were treated by complete cyst resection, lung cysts $(83.3 \%)$ were predominantly treated by cyst resection plus capitonnage. Pathological examination revealed fertile cysts in $24 \%$. Postsurgical morbidity was frequent (37.9\%), and $13.3 \%$ of the series required readmission for this cause. Near $25 \%$ of patients remained in hospital $>14$ days, a feature associated with fever during admission $(\mathrm{p}<0.05)$. No recurrence was reported in the fraction of patients that were followed-up for 2 years or more. Conclusions: Our analysis of 23 cases demonstrates that patients were mainly adults suffering from abdominal cysts. Most patients were successfully treated by surgery with or without antiparasitic drugs but complications leading to prolonged hospital stays and readmissions were not infrequent.

Key words: Echinococcus granulosus, hepatic hydatid cyst, pulmonary hydatid cyst, diagnosis, therapeutics, pericystectomy, cystostomy, capitonnage, albendazole, pathology.

Palabras clave: Echinococcus granulosus, quiste hidatídico hepático, quiste hidatídico pulmonar, diagnóstico, tratamiento, pericistectomía, quistostomía capitonaje, albendazol, patología.

\section{Introducción}

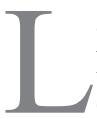
a equinococcosis quística, conocida también como hidatidosis, es la enfermedad producida por el estado larval de Echinococcus granulosus. Se caracteriza por la presencia de un quiste, habitualmente hepático, en segundo lugar pulmonar y también en forma combinada o en otras localizaciones. Los quistes pueden provocar síntomas por ocupación de tejidos o sus complicaciones. Esta zoonosis parasitaria se adquiere por la ingestión de huevos de E. granulosus excretados en las deposiciones del perro, que contaminan agua o alimentos y que también están presentes en su pelaje. El ciclo de transmisión se establece habitualmente en relación a la crianza de ganado bovino, ovino o caprino, el que desarrolla la infección como hospedero intermediario, con formación de quistes viscerales y donde el ciclo se perpetúa por el uso de estas visceras en la alimentación del perro luego de sacrificarse el ganado. El hombre es un hospedero accidental intermediario en este ciclo ${ }^{1}$ y la infección es favorecida, entre otros factores, por la ignorancia, analfabetismo, ruralidad, cercanía con actividades ganaderas, beneficio clandestino de animales, uso de las vísceras para alimentar perros y ausencia de programas de intervención ${ }^{2}$. La hidatidosis humana asociada a $E$. granulosus es una enfermedad desatendida, de amplia distribución y que se encuentra presente en Eurasia, África, Australia y Sudamérica. Representa una causa perceptible de morbilidad, intervenciones quirúrgicas, egresos hospitalarios, gastos médicos y ocasionalmente puede provocar la muerte ${ }^{1}$. En Chile, se han desarrollado exitosos programas de control de la hidatidosis en el extremo austral, iniciados en la década del $70^{1,3}$. Sin embargo, mantenemos una situación endémica con cifras que no descienden en las últimas décadas y se mantienen cerca de dos casos por 100.000 habitantes con claras evidencias de sub-notificación, cifras reales probables de 10 por 100.000 habitantes $^{4,5} \mathrm{y}$ con algunos sectores en el norte del país donde las tasas de seroprevalencia sobrepasan el $1 \%$ de la población ${ }^{3,6,7}$ lo que ilustra la gran importancia
Hospital Militar de Santiago, Chile.

Departamento de Medicina Servicio de Infectología (AF, AS). Servicio de Anatomía Patológica (JS).

Departamento de Cirugía (MP $\mathrm{RH}, \mathrm{JG}$ ).

Servicio de Imagenología (MC). Clínica Alemana/Universidad Del Desarrollo, Santiago, Chile (TW)

Conflicto de interés: ninguno. Fuente de financiamiento: ninguna.

Recibido: 29 de junio de 2011 Aceptado: 8 de noviembre de 2011

Correspondencia a: Alberto Fica Cubillos albertofica@gmail.com. 
epidemiológica de este problema ${ }^{7,8}$. Su dependencia con la crianza de ganado hace que esta enfermedad se presente en forma focalizada pero la movilidad de la población por migración o viajes la transforma en problema clínico en varios centros de hospitalización donde la experiencia es por lo tanto disímil. Este trabajo expone nuestra experiencia en casos de hidatidosis humana durante 15 años en un hospital urbano, analizando las diferentes formas de presentación clínica, los hallazgos histológicos, las formas de tratamiento y la evolución de los casos.

\section{Métodos}

Diseño del estudio y pacientes. Estudio retrospectivo descriptivo con una serie de pacientes identificados utilizando tres estrategias: por egresos hospitalarios (código B67.0 de la Clasificación Internacional de Enfermedades CIE 10 disponible desde el año 1998), a través de la base de datos de la actividad quirúrgica del Departamento de Cirugía (1996 en adelante) y por el registro de farmacia de los tratamientos con albendazol (2005 en adelante). Algunos pacientes fueron hospitalizados más de una vez para ser tratados quirúrgicamente en forma secuencial pero siempre en el mismo año, generando más casos (hospitalizaciones quirúrgicas) que pacientes. Se incluyeron todos los casos detectados desde el año 1996 al 2010. No hubo acceso a registros más antiguos.

Caracterización clínica. Se analizaron datos demográficos, antecedentes mórbidos, manifestaciones clínicas, estudio de imágenes, residencia o visitas a áreas endémicas para hidatidosis (Región de Coquimbo en el norte y desde Región del Maule a Región de Magallanes en el sur de Chile), pruebas de laboratorio, incluyendo eosinófilos en sangre, estudio serológico, tratamiento quirúrgico y médico antiparasitario. Las variables demográficas, epidemiológicas, las manifestaciones clínicas, los datos de laboratorio, de imágenes y los tratamientos antiparasitarios fueron expresados por número de pacientes ya que no hubo un lapso de tiempo prolongado entre las hospitalizaciones quirúrgicas que justificara colocarlas por separado. En el tratamiento médico se precisó si hubo uso de fármacos antiparasitarios, antes o después de la cirugía. En el caso del uso pre-quirúrgico sólo se consignó este dato por una vez, independientemente a si el paciente tuvo dos o más intervenciones quirúrgicas. Las dosis fueron expresadas por kilo de peso y se calculó el número de ciclos de tratamiento asumiendo arbitrariamente como ciclo un período de cuatro semanas. Para el tratamiento quirúrgico se precisó si hubo abordaje por toracotomía, laparotomía, toracoscopia o laparoscopia, procedimientos de punción-aspiración antes de la extirpación, uso de sustancias escolicidas y el manejo de la cavidad (capi- tonaje). En cirugía hepática los procedimientos fueron catalogados como conservadores cuando no se extrajo el quiste dejando una cavidad residual o alternativamente, como radicales cuando el quiste fue extirpado. En este último caso se precisó si se efectuó resección segmentaria o periquistectomía. En los pacientes con cirugía torácica se clasificó la cirugía como quistostomía más capitonaje o como resección segmentaria pulmonar. Además, se consignó la evolución del paciente como, complicada o no complicada, de acuerdo a la aparición de eventos post-operatorios (cualquiera). Por otra parte, se consideró como quiste complicado aquel con evidencias de rotura en la cirugía o con evidencias de conexión con la vía aérea o biliar en los exámenes pre-operatorios, independientemente a si la evolución del paciente fuese complicada o no. Estas variables fueron expresadas por el número de hospitalizaciones quirúrgicas.

Anatomía patológica. El material de biopsia fue revisado para identificar la presencia de adventicia, cutícula, prolígera (membrana proliferativa), cápsulas germinativas, protoescólices, ganchos o vesículas hijas ${ }^{9}$. Los quistes además fueron identificados como fértiles o estériles ${ }^{1}$.

Análisis estadístico. Las variables categóricas fueron comparadas en tablas de contingencia por la prueba de $\chi^{2}$ o bilateral de Fisher con un nivel de significación $\mathrm{p}<$ 0,05 . Algunas variables continuas fueron transformadas en variables categóricas y sometidas al mismo proceso. Las tendencias de casos en el tiempo fueron analizadas por el coeficiente de correlación de Pearson.

\section{Resultados}

Pacientes y datos demográficos. Se identificaron 23 pacientes que requirieron 30 hospitalizaciones quirúrgicas en el período y todos fueron intervenidos una o dos veces entre los años 1996 y 2010. La edad promedio fue de 38,8 años (mediana 40 años; rango 5 a 73). La mayor parte de los pacientes $(73,9 \%)$ tenía menos de 50 años (Tabla 1) y $13 \%$ tenían menos de 15 años. No hubo diferencias significativas en la distribución por sexo. En 76,5\% de los pacientes con registro disponible había antecedentes de residencia o visita a regiones endémicas para hidatidosis. Los casos se presentaron con una frecuencia variable entre 1 y 6 eventos por año con una tendencia no significativa al descenso (coeficiente de Pearson -0,37, datos no mostrados). Un paciente tenía antecedentes de otro caso de hidatidosis en la familia (4,3\% del total de pacientes) sin figurar este antecedente en el resto de los registros y en sólo un caso se efectuó estudio al resto de los integrantes de la familia $(4,5 \%)$. 
Localización. En 19 de los 23 pacientes se hicieron estudios complementarios de diseminación $(82,6 \%)$ antes de la cirugía, ya fuese con ecotomografía abdominal, radiografías de tórax o estudios tomográficos computarizados. La mayor parte de los quistes se ubicó en la región abdominal, en forma aislada o combinada (18 de $23 ; 78,3 \%$ ). Cerca de la mitad de los pacientes tenía dos o más quistes (Tabla 1). El diámetro mínimo de los quistes se ubicó entre los 1 y $3 \mathrm{~cm}$ y el diámetro máximo entre los 10 y $20 \mathrm{~cm}$ pero el promedio del diámetro del segundo o tercer quiste fue similar al del primer quiste (Tabla 1). Un total de 7 quistes tenía signos de calcificación y todos ellos en ubicación hepática $(\mathrm{p}<0,05$ por prueba bilateral de Fisher).

Presentación clínica. Cerca de tres cuartas partes de los pacientes acusaban síntomas respiratorios o abdominales y el resto fue pesquisado por exámenes de rutina (Tabla 1). Más de 70\% de los pacientes que tenían quistes en la cavidad abdominal presentaron síntomas y el dolor abdominal fue el síntoma más frecuente de los reportados (Tabla 1). Ningún paciente se presentó con ictericia (Tabla 1). El $60 \%$ de los pacientes con quistes en la cavidad torácica refirió síntomas (6 de 10), los más frecuentes fueron el dolor torácico y la tos $(83,3 \%$ del subgrupo de pacientes con síntomas, Tabla 1). La vómica fue detectada en tres de estos pacientes. Los síntomas obstructivos bronquiales estuvieron presentes en al menos uno de cada tres pacientes sintomáticos. Tanto la fiebre como la diaforesis fueron pesquisadas en algunos pacientes pero con baja frecuencia. En esta serie, la fiebre no se asoció a quistes complicados ( $p>0,05$ por prueba bilateral de Fisher). La mayor parte de los pacientes $(80 \%)$ se presentó sin anemia o leucocitosis, aunque fue posible detectar casos con estas condiciones (Tabla 2). Cerca de $40 \%$ presentó > $5 \%$ de eosinófilos o recuentos sobre $500 / \mathrm{mm}^{3}$ y una cifra cercana a $25 \%$ tuvo recuentos $>1.000 / \mathrm{mm}^{3}$. El promedio fue $709 / \mathrm{mm}^{3}$ (rango 0 a 4.554). La eritrosedimentación promedio fue de $29,5 \mathrm{~mm} / \mathrm{h}$ (rango 1-105) y cerca de $40 \%$ sobrepasó el valor normal (Tabla 2). Los tres pacientes que presentaron valores sobre $90 \mathrm{~mm} / \mathrm{h}$ tuvieron fiebre como antecedente ( $\mathrm{p}<0,01$ por prueba bilateral de Fisher). El estudio serológico (ELISA IgG) fue solicitado en no más de $60 \%$ de los afectados y la positividad fue de $57,1 \%$. En esta serie no se encontró una asociación entre serología positiva y la edad del paciente, el recuento de eosinófilos, número de quistes, ubicación del quiste, ausencia de calcificaciones o quistes complicados (datos no mostrados). Para el subgrupo con hidatidosis hepática, la mayor parte no presentó aumento de la bilirrubinemia ( $>90 \%$ normal) o de aminotransferarasas ( $>75 \%$ normal) pero más de la mitad presentó un valor elevado de $\gamma$-glutamil transpeptidasa (promedio $178 \mathrm{UI} / \mathrm{L}$, rango 6 a 646) y especialmente de fosfatasas alcalinas $(84,6 \%$; promedio 143,8, rango 64 a $474 \mathrm{UI} / \mathrm{L})$ (Tabla 2).

Tratamiento antiparasitario. Cerca de $60 \%$ de los pacientes recibió tratamiento antiparasitario el que siempre fue efectuado con albendazol. Los tratamientos fueron prescritos por 1 a 6 meses y la mitad de los pacientes

Tabla 1. Características demográficas y clínicas en 23 pacientes con hidatidosis

\begin{tabular}{|c|c|c|}
\hline Características generales & $\mathrm{n}^{*} / \mathrm{N}^{+}$ & $\%$ \\
\hline Edad $<50$ años & $17 / 23$ & 73,9 \\
\hline Edad $<15$ años & $3 / 23$ & 13,0 \\
\hline Sexo masculino & $13 / 23$ & 56,5 \\
\hline Residencia o visitas a área endémica & $13 / 17$ & 76,5 \\
\hline \multicolumn{3}{|l|}{ Ubicación de los quistes sobre el total de pacientes } \\
\hline Abdominal & $18 / 23$ & 78,3 \\
\hline Sólo hepático & $12 / 23$ & 52,2 \\
\hline Hepato-pulmonar & $5 / 23$ & 21,7 \\
\hline Hepato-esplénico & $1 / 23$ & 4,3 \\
\hline Pulmonar & $5 / 23$ & 21,7 \\
\hline \multicolumn{3}{|l|}{ Número de quistes por paciente } \\
\hline Uno & $13 / 23$ & 56,5 \\
\hline Dos & $5 / 23$ & 21,7 \\
\hline Tres & $3 / 23$ & 13,0 \\
\hline$>3$ & $2 / 23$ & 8,8 \\
\hline Diámetro máximo en $\mathrm{cm}$ & Promedio & Rango \\
\hline Quiste único & 7,7 & $3-20$ \\
\hline Segundo quiste & 7,0 & $1,2-15,0$ \\
\hline Tercer quiste & 7,3 & 1,5 a 10,7 \\
\hline Características clínicas & $n^{*} / N^{+}$ & $\%$ \\
\hline Presencia de síntomas & $17 / 23$ & 73,9 \\
\hline Síntomas abdominales en pacientes con quistes abdominales & $10 / 14$ & 71,4 \\
\hline Dolor abdominal & $8 / 10$ & 80,0 \\
\hline Molestias vagas & $4 / 10$ & 40,0 \\
\hline Vómitos o náuseas & $2 / 10$ & 80,0 \\
\hline Ictericia & $0 / 10$ & 0,0 \\
\hline Síntomas respiratorios en pacientes con quistes torácicos & $6 / 10$ & 60,0 \\
\hline Dolor torácico & $5 / 6$ & 83,3 \\
\hline Tos & $5 / 6$ & 83,3 \\
\hline Disnea & $4 / 6$ & 66,7 \\
\hline Vómica & $3 / 6$ & 50,0 \\
\hline Obstrucción bronquial & $2 / 6$ & 33,3 \\
\hline \multicolumn{3}{|l|}{ Síntomas sistémicos } \\
\hline Fiebre & $5 / 23$ & 21,7 \\
\hline Diaforesis & $1 / 23$ & 4,3 \\
\hline
\end{tabular}


Tabla 2. Parámetros de laboratorio en hidatidosis. Hospital Militar 1996-2010

\begin{tabular}{lcc} 
Variables & $\mathrm{n} / \mathrm{N}$ & $\%$ \\
Hemoglobina $<12 \mathrm{~g} / \mathrm{dL}$ & $4 / 20^{*}$ & 20,0 \\
Leucocitosis $>11.000 / \mathrm{mm}^{3}$ & $4 / 20^{*}$ & 20,0 \\
\hline Eosinófilos $>5 \%$ o recuento de eosinófilos $>500 / \mathrm{mm}^{3}$ & $7 / 17^{*}$ & 41,2 \\
Recuento de eosinófilos $>1.000 / \mathrm{mm}^{3}$ & $4 / 17^{*}$ & 23,5 \\
\hline Eritrosedimentación $>20 \mathrm{~mm} / \mathrm{h}$ & $8 / 19^{*}$ & 42,1 \\
\hline Estudio serológico de hidatidosis solicitado & $14 / 23$ & 60,9 \\
\hline Estudio serológico positivo & $8 / 14^{*}$ & 57,1 \\
\hline En pacientes con quistes hepáticos: & $1 / 14^{\dagger}$ & 7,1 \\
\hline Bilirrubinemia total $>1 \mathrm{mg} / \mathrm{dL}$ & $3 / 14^{\dagger}$ & 21,4 \\
\hline Aminotransferasas $>$ valor normal & $4 / 7^{\dagger}$ & 57,1 \\
\hline$\gamma$-glutamil transpeptidasa $>$ valor normal & $11 / 13$ & 84,6 \\
\hline Fosfatasas alcalinas $>$ valor normal & & \\
\hline *sobre pacientes con datos disponibles; ${ }^{\dagger}$ sobre pacientes con quistes hepáticos y datos disponibles.
\end{tabular}

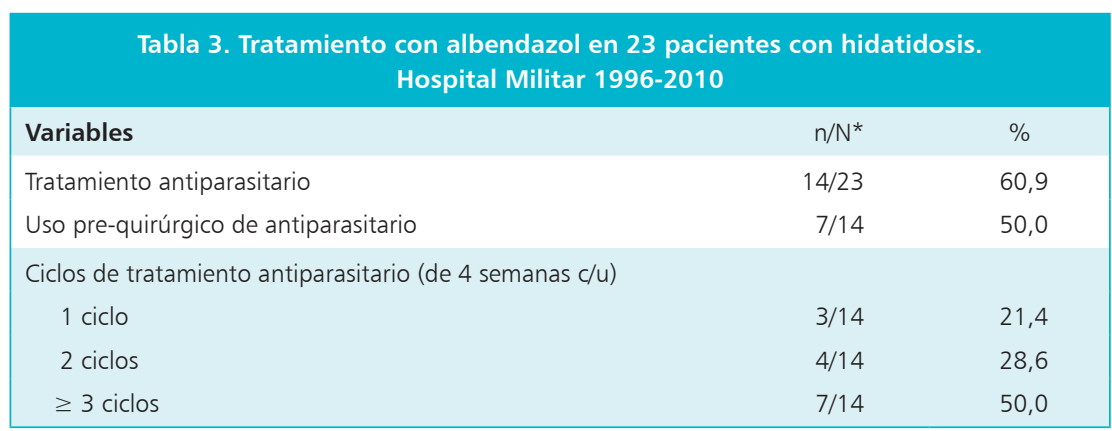

Tabla 4. Tratamiento quirúrgico de la hidatidosis. Hospital Militar 1996-2010

\begin{tabular}{lcc} 
Variables & $\mathrm{n} / \mathrm{N}^{*}$ & $\%$ \\
Quistes complicados & $6 / 30$ & 20,0 \\
Sitio operado & $18 / 30$ & 60,0 \\
$\quad$ Hepático & $12 / 30$ & 40,0 \\
$\quad$ Pulmonar & & \\
Cirugía hepática & $2 / 18$ & 11,1 \\
$\quad$ Conservadora & $16 / 18$ & 88,9 \\
$\quad$ Radical & $4 / 18$ & 22,2 \\
$\quad$ Resección & $12 / 18$ & 66,7 \\
$\quad$ Periquistectomía & & \\
Cirugía pulmonar & $2 / 12$ & 16,7 \\
$\quad$ Resección por segmentectomía & $10 / 12$ & 83,3 \\
$\quad$ Quistostomía más capitonaje con cierre de aperturas bronquiales & & \\
Otros aspectos quirúrgicos & $14 / 29^{*}$ & 48,3 \\
$\quad$ Punción y aspiración del quiste & $10 / 29^{*}$ & 34,5 \\
$\quad$ Uso de solución escolicida & $10 / 24^{\dagger}$ & 41,7 \\
$\quad$ Manejo de la cavidad & & \\
\hline *sobre tratamientos con datos disponibles; ${ }^{\dagger}$ sobre casos sin resección. & & \\
\hline
\end{tabular}

recibió al menos tres ciclos (Tabla 3). La dosis promedio de albendazol fue $11,7 \mathrm{mg} / \mathrm{kg}$ / día (rango 6 a $17 \mathrm{mg} / \mathrm{kg}$ / día). En siete casos se usó albendazol antes de la cirugía, por un promedio de 7,4 días (rango 2 a 28). El diseño retrospectivo del estudio no permitió evaluar la aparición de efectos adversos asociados a albendazol y sólo se registró un caso de alopecia que obligó a suspender su uso después del alta.

Tratamiento quirúrgico y condición de los quistes. Veinte por ciento de los quistes operados estaba complicado, tres hepáticos y tres pulmonares (Tabla 4). Las Figuras 1 a 3 muestran ejemplos de quistes complicados y no complicados hepático o pulmonar. Todos los pacientes fueron operados por toracotomía o laparotomía y no se detectaron casos manejados por procedimientos percutáneos radiológicos. La mayor parte de los pacientes con quistes hepáticos fueron operados con un procedimiento radical $(88,9 \%)$ siendo la periquistectomía el procedimiento más frecuente (12 de 18 intervenciones hepáticas, Tabla 4) secundada por una resección segmentaria hepática en otros cuatro casos $(22,2 \%)$. En dos casos se aplicó un procedimiento conservador con quistostomía y extracción de la membrana prolígera $(11,1 \%)$. La mayor parte de las cirugías en quistes pulmonares fue realizada con una remoción del quiste por quistostomía con capitonaje de la cavidad y cierre de las aperturas bronquiales (Tabla 4) y en sólo dos casos se realizó una resección segmentaria. En términos globales, en aproximadamente la mitad de los casos se aplicó una aspiración y punción del quiste, en un tercio se uso una sustancia escolicida directamente en la cavidad (siempre como solución hipertónica al 30\%) y en $\sim 42 \%$ de los casos donde se extrajo el quiste, se efectuó capitonaje de la cavidad residual (Tabla 4).

Evolución y seguimiento. Cerca de $40 \%$ de los casos operados evolucionó con complicaciones (Tabla 5). Estas complicaciones fueron observadas en 11 eventos quirúrgicos, cinco de ellas en quistes pulmonares (42\% morbilidad) y el resto en cirugías de quistes hepáticos (33\% morbilidad). El tipo de complicación no fue uniforme e incluyó para el primer grupo atelectasias, neumonía, neumotórax o fístula bronquial, y para el segundo grupo, anemia con requerimiento transfusional, colecciones post-quirúrgicas, celulitis de la pared abdominal y fístula biliar. En cuatro de estas 10 complicaciones hubo que re-hospitalizar al paciente para su manejo. En esta serie, el número total del re-hospitalizaciones fue de 10 , correspondiendo cuatro de ellas al manejo de las complicaciones ya mencionadas (13,3\% del total de hospitalizaciones) y otras seis, a la cirugía en un segundo tiempo de otro quiste hidatídico. La estadía promedio fue de 12,5 días (rango 4 a 52 días). Cerca de un cuarto de los pacientes tuvo estadías mayor a 14 días y este rasgo se asoció sig- 


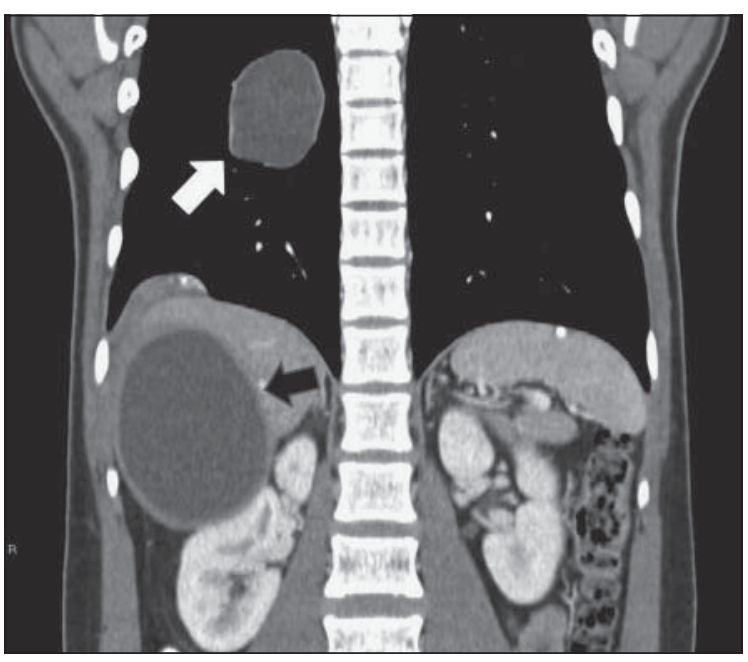

Figura 1. Reconstrucción coronal de una tomografía computada multicorte que revela un quiste pulmonar derecho (flecha blanca) y un quiste hepático (flecha negra) de paredes gruesas y sin evidencia de complicaciones.

nificativamente a la presencia de fiebre al ingreso (estadía $\geq$ a 14 días en 4 de 5 casos con fiebre vs 3 de 17 casos sin fiebre; $p<0,05$ por prueba bilateral de Fisher), pero no a otros factores. Los pacientes fueron seguidos por un promedio de 2,9 años después de cada cirugía (rango 0 a 13,7 años) pero sólo $41 \%$ de ellos fue contralado más allá de dos años (Tabla 5) y en ninguno de estos casos se registró una recurrencia de la enfermedad. Cuatro casos de la serie $(14,3 \%)$ se perdieron después del alta. En dos casos adicionales se detectó un quiste residual $(6,9 \%)$. No se registraron decesos en esta serie.

Hallazgos histológicos. En 25 de los 30 quistes operados se pudo acceder al material de biopsia para revisar las características de los quistes extirpados. La distribución de los hallazgos se presenta en la Tabla 6 donde se aprecia el predominio de la adventicia (reacción del hospedero) y de la cutícula (pared del parásito). En $24 \%$ de los casos las biopsias demostraron quistes fértiles (presencia de protoescólices, ganchos, vesículas o cápsulas prolígeras, Figuras 4 a 6). La presencia de un quiste fértil no se asoció significativamente al resultado de la serología, al valor de la eritrosedimentación, a la magnitud de la esosinofilia, a la edad del paciente, a la presencia de fiebre al ingreso o al uso pre-quirúrgico de albendazol. No obstante, la fertilidad se asoció significativamente a quistes pulmonares y no a quistes hepáticos ( 5 de 11 pulmonares eran fértiles vs 1 de 14 hepáticos, p: 0,026). Por otra parte, ninguno de los cinco quistes calcificados fue fértil en contraste con 6 de los 20 quistes no calcificados, aunque estas diferencias no alcanzaron a ser significativas por el bajo tamaño de la muestra.
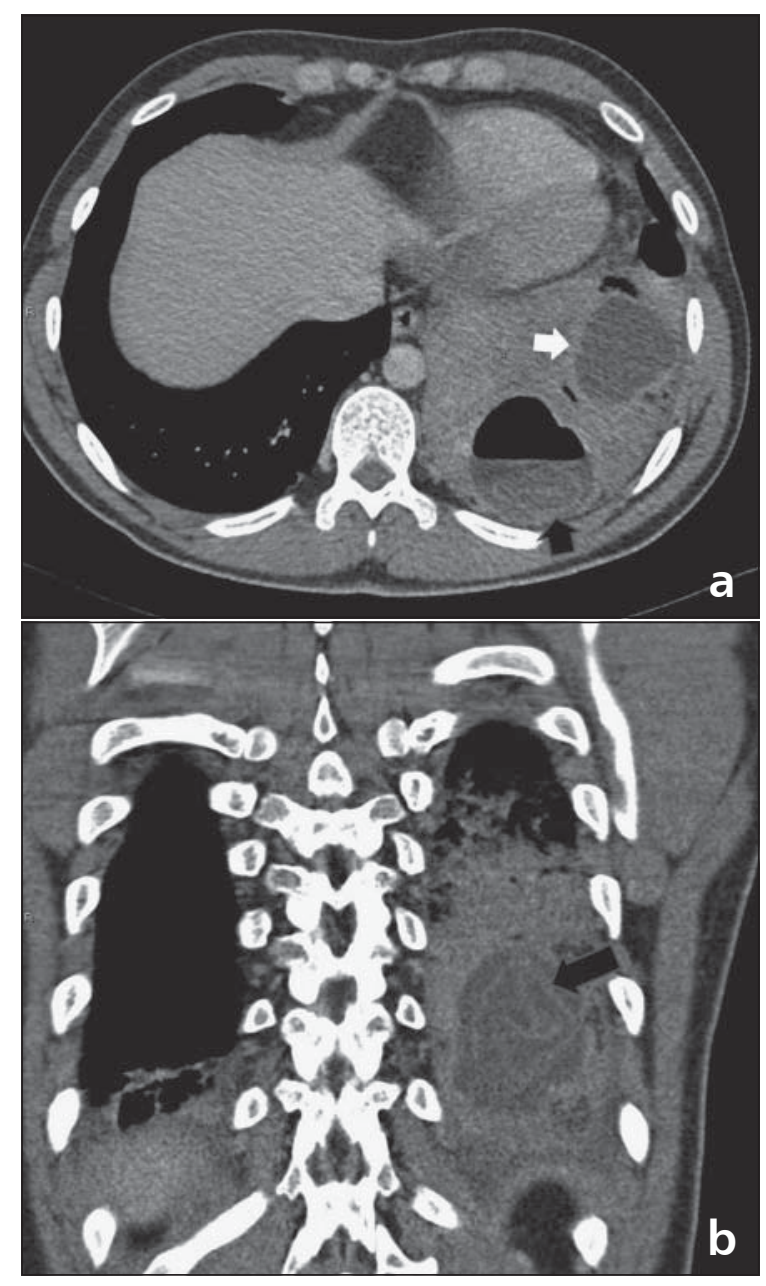

Figura 2. a. Corte axial de una tomografía computada multicorte que demuestra un quiste hidatídico pulmonar izquierdo no complicado (flecha blanca) y otro comunicado a árbol bronquial, presentando un nivel hidroaéreo y membranas en su interior (flecha negra). b. El mismo paciente en una reconstrucción coronal donde se aprecian las membranas con mayor claridad (flecha negra).

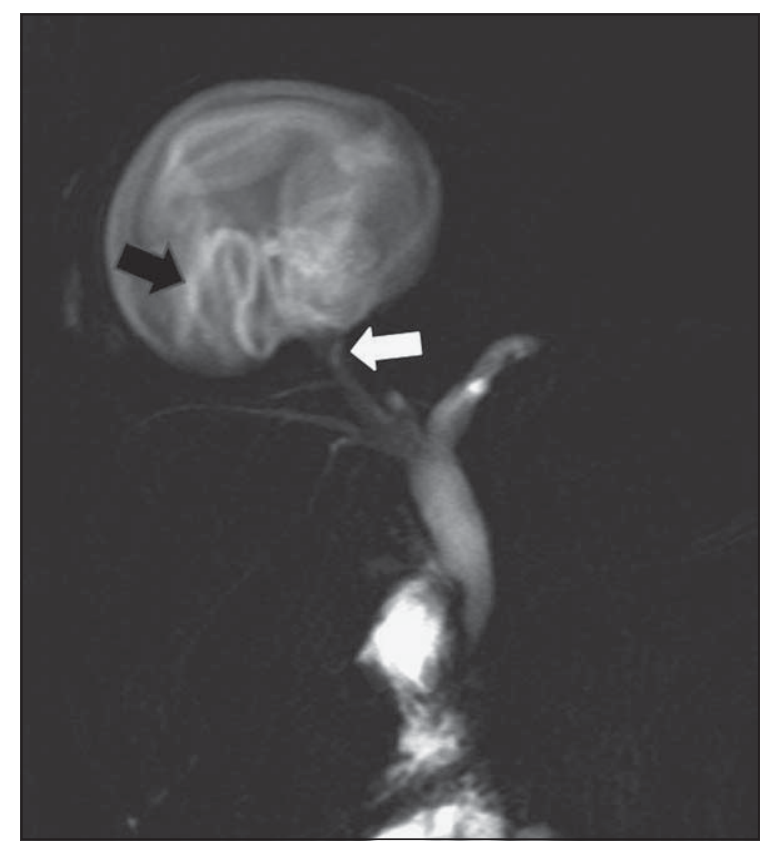

Figura 3. Adquisición volumétrica por resonancia magnética que revela un quiste hidatídico hepático comunicado con la vía biliar (flecha blanca). Se observan membranas al interior del quiste (flecha negra) y un conducto biliar prominente comunicado al resto del árbol biliar. 
Figura 4. Tinción de GomoriGrocott \& hematoxilina-eosina x400. Se aprecia protoescólex invaginado $(P)$, rostelo con ganchitos (R) y en la parte superior cutícula multilaminar (C) y membrana prolígera (flecha).

Figura 5. Tinción hematoxilina-eosina $\times 200$. Vesículas prolígeras.

Figura 6. Tinción hematoxilina-eosina x200. Cutícula multilaminar (C), membrana prolígera $(\mathrm{M})$ y protoescólex $(\mathrm{P})$.
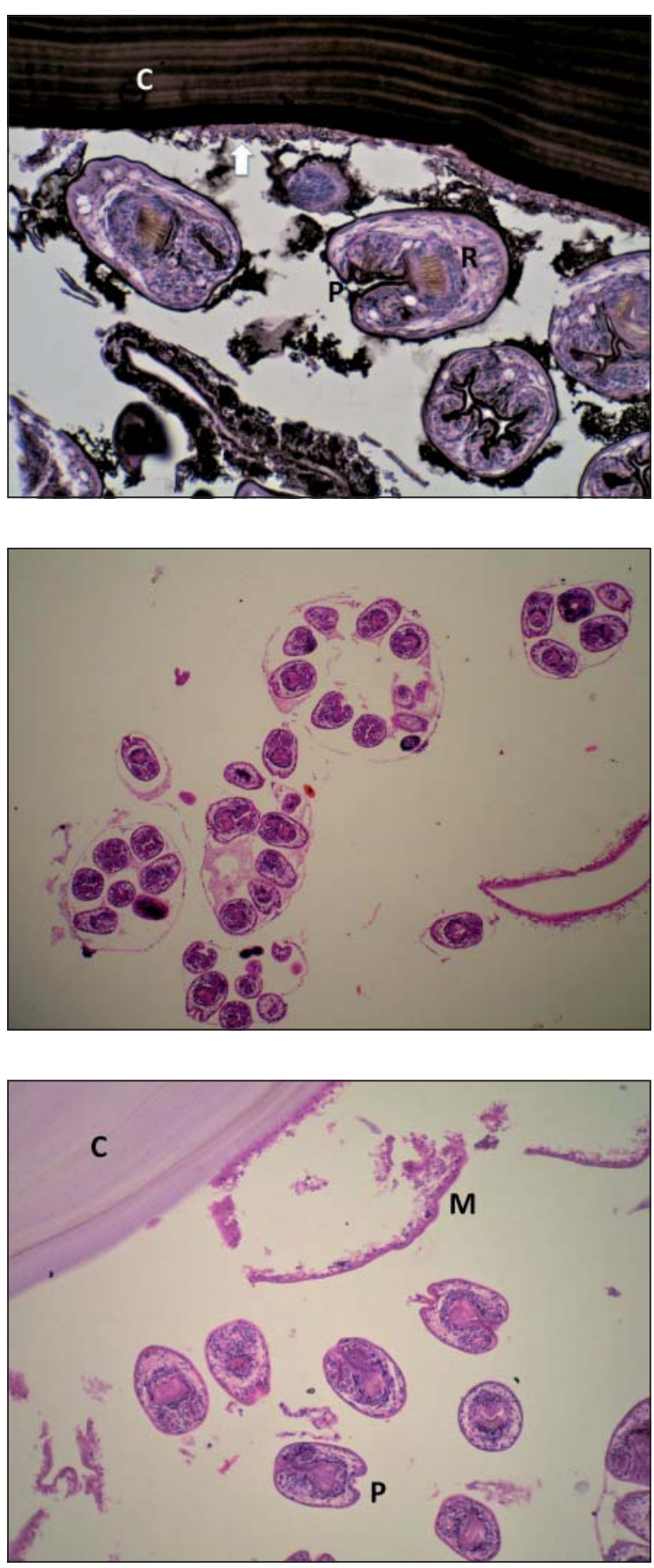

\section{Discusión}

Los resultados de este trabajo confirman la persistencia de esta histoparasitosis en un solo centro hospitalario ubicado fuera de las regiones endémicas en Chile. La infección afectó preferentemente a una población joven con potencial productivo ${ }^{2}$. El grupo de pacientes que
Tabla 5. Evolución y seguimiento de 23 pacientes con

hidatidosis (30 hospitalizaciones). Hospital Militar. 1996-2010

\begin{tabular}{|lcc|} 
Parámetros & $\mathbf{n} / \mathbf{n}$ & $\%$ \\
Evolución con complicaciones & $11 / 29^{*}$ & 37,9 \\
$\begin{array}{l}\text { Rehospitalizaciones por } \\
\text { complicaciones }\end{array}$ & $4 / 30$ & 13,3 \\
Estadía > 14 días & $7 / 29^{*}$ & 24,1 \\
Seguimiento > 2 años & $12 / 29^{*}$ & 41,4 \\
$\begin{array}{l}\text { Recurrencia en pacientes con } \\
\text { seguimiento > 2 años }\end{array}$ & $0 / 12$ & 0,0 \\
Quiste residual & $2 / 29^{*}$ & 6,9 \\
\hline *sobre tratamientos con datos disponibles. & \\
\hline
\end{tabular}

Tabla 6. Hallazgos histológicos en 25 biopsias de quistes hidatídicos Hospital Militar. 1996-2010

\begin{tabular}{lcc|} 
Parámetro & $\mathbf{n}$ & $\%$ \\
Adventicia & 23 & 92,0 \\
Cutícula & 21 & 84,0 \\
Membrana prolígera & 14 & 56,0 \\
Protoescólices & 5 & 20,0 \\
Cápsulas germinales & 2 & 8,0 \\
Vesículas prolígeras (sin cutícula) & 2 & 8,0 \\
Ganchos & 3 & 12,0 \\
Total de quistes fértiles* & 6 & 24,0 \\
\hline
\end{tabular}

*incluye protoescólices, ganchos, vesículas prolígeras o cápsulas germinales.

refería residir o visitar zonas endémicas no participaba en actividades ganaderas o agrícolas, subrayando el desplazamiento de los grupos tradicionales de riesgo ${ }^{2}$ hacia una población urbana por fenómenos de migración y visitas a sus sitios de origen. Este fenómeno puede limitar la sospecha clínica ya que el lugar de exposición puede estar disociado del lugar donde el paciente consulta varios años después por la lenta progresión de los quistes. Por otra parte, en al menos un caso se comprobó la existencia de otro caso familiar, a pesar de que no hubo un estudio sistemático para ello. El acumulo de casos familiares ha sido previamente descrito y sugiere que los contactos familiares deberían ser estudiados en forma activa ${ }^{10}$ Nuestros datos confirman también la baja letalidad de la hidatidosis humana pero no se pudo verificar una tendencia al descenso de los casos, un fenómeno compatible con las cifras estables de incidencia reportada en Chile 4 .

Las manifestaciones clínicas de la hidatidosis son inespecíficas pero algunos elementos permiten su sospecha como la vómica o la eosinofilia asociada a la presencia de 
una imagen quística en el hígado o el pulmón mediante estudio de imágenes. La baja positividad de las pruebas serológicas encontradas en este trabajo es concordante con la baja sensibilidad del inmunodiagnóstico para esta enfermedad ${ }^{11,12}$. El diagnóstico inmunológico de la hidatidosis adolece de problemas de sensibilidad y especificidad que aún no han sido resueltos ${ }^{13}$. Las técnicas de mayor rendimiento están basadas en pruebas de inmunoelectroforesis (detección del arco 5 de Caprón en doble difusión en agar), ELISA e inmunoblotting, las que tienen una precisión variable por la naturaleza del antígeno utilizado y su forma de purificación ${ }^{11,14}$. Incluso, el uso de antígenos recombinantes no ha logrado resolver del todo estas limitaciones ${ }^{13}$. Las deficiencias en especificidad son explicadas por las reacciones cruzadas con otras parasitosis ${ }^{13}$. De esta manera, el diagnóstico de hidatidosis depende de la mejor conjunción de datos epidemiológicos, clínicos, imagenológicos y serológicos y, cada vez que se pueda, debe ser confirmado por el estudio histológico o el análisis del contenido del quiste ${ }^{9,15}$. Como se observó, la fiebre junto a una eritrosedimentación muy elevada no descarta la hidatidosis humana.

Actualmente existen tres opciones de tratamiento que corresponden a la cirugía, punción percutánea bajo guía imagenológica y el tratamiento con fármacos antiparasi$\operatorname{tarios}^{1}$. La opción de una u otra modalidad depende de la experiencia del grupo tratante, del número y localización de los quistes, de la existencia de complicaciones asociadas, de la evidencia acumulada en la literatura médica y de las preferencias del paciente. En esta serie, el tratamiento fue esencialmente quirúrgico; no se observaron casos tratados por punción percutánea o por laparoscopia/videotoracoscopia. Cerca de $90 \%$ de nuestros pacientes con quistes hepáticos fueron operados con una cirugía radical y sólo en dos (11\%) se aplicó un criterio conservador. Esta preferencia obedece a la gran eficacia observada $(\sim 100 \%)$ cuando se logra la extirpación completa de la membrana germinativa y no se dejan cavidades residuales ni comunicaciones con el exterior ${ }^{16}$. Los resultados no son tan espectaculares en el caso de las cirugías conservadoras. Las ventajas de la cirugía radical respecto a su contraparte han sido establecidas en diferentes series de pacientes y al menos en un ensayo controlado que demuestran como esta estrategia permite una menor recidiva local y se asocia además a una menor tasa de morbilidad post-operatoria y complicaciones hepáticas ${ }^{17}$. La cirugía radical contempla la periquistectomía y en otros casos, según las condiciones locales, una segmentectomía o lobectomía. En contraste, la cirugía conservadora consiste en el drenaje de la cavidad con una remoción parcial de las membranas y fue históricamente la aproximación más frecuente por su facilidad y menor tiempo quirúrgico ${ }^{16,18}$ pero está asociada a un mayor número de complicaciones tales como fístula biliar, absceso de la cavidad ${ }^{17,18} \mathrm{o}^{\text {mortalidad }^{12}}$. A pesar de las ventajas de la cirugía radical hepática, ésta no puede ser aplicada en todos los casos ya que requiere una infraestructura mayor, mejor entrenamiento quirúrgico y es difícil de aplicar en algunos pacientes con quistes profundos asociados a estructuras vasculares, y en algunos de los que se presentan con una comunicación biliar ${ }^{12,17}$. La alta frecuencia de cirugía radical en nuestro centro contrasta con los bajos porcentajes observados en otras ciudades del país ( $<10 \%$ en la Región del Maule, una zona endémica), subrayando las variaciones en las preferencias y posibilidades terapéuticas en un mismo país ${ }^{18}$. A diferencia de la resección del tejido circundante en los quistes hepáticos, en la cirugía pulmonar no se remueve la zona periquística por el riesgo de desarrollar fístulas broncopleurales. En su lugar se abre el quiste y se extirpa su contenido con la prolígera y cutícula. Este procedimiento se denomina quistostomía más capitonaje con cierre de aperturas bronquiales, ya que incorpora el manejo de la cavidad residual con esta técnica ${ }^{19} \mathrm{y}$ tiene una alta eficacia terapéutica con $<2 \%$ recurrencia a largo plazo $^{20}$. Fue el procedimiento predominante en nuestros pacientes. El cierre de las aperturas bronquiales y el capitonaje de la cavidad evita además, empiemas y colecciones. Otra de sus ventajas es su menor estadía hospitalaria. La resección del tejido pulmonar es necesaria en la fracción restante cuando hay gran destrucción tisular o la reconstrucción de la vía aérea es imposible ${ }^{19,20}$. En grandes series, la mortalidad de la cirugía pulmonar es $<1,5 \%$ y su tasa de morbilidad varía entre 0 y $17 \%{ }^{19}$ dependiendo de la definición utilizada. La tasa de morbilidad global en cirugía hepática o pulmonar alcanzó a $42 \%$ en nuestra serie al considerar todo tipo de complicaciones. De esta manera, los mejores enfoques quirúrgicos actuales no evitan que en algunos pacientes la estadía hospitalaria sea prolongada o que tengan reingresos, lo que subraya la carga de enfermedad que representa esta histoparasitosis. En el caso de quistes múltiples donde no se pueden identificar todas las lesiones, existe el riesgo de una enfermedad residual como fue observado en nuestros casos.

En el presente trabajo se observó una asociación significativa entre fertilidad y quistes pulmonares y al mismo tiempo, la ausencia de calcificación en los quistes en este compartimento ${ }^{1,21}$.

No más de $60 \%$ de nuestros pacientes recibió terapia concomitante con albendazol, que persigue reducir el riesgo de recurrencia ${ }^{22}$. A pesar de que no existen estudios comparativos directos entre albendazol y cirugía, se considera que la farmacoterapia es una opción secundaria a la cirugía ya que con la opción médica, no más de $50 \%$ de los pacientes demuestra una desaparición de sus quistes, al menos 20 a $25 \%$ no muestra variaciones en su tamaño y cerca de 15 a $30 \%$ presenta recurrencias durante el seguimiento ${ }^{22-25}$. El tratamiento requiere cursos prolongados de al menos tres meses con albendazol ${ }^{23,24,26,27}$. Las indicacio- 
nes clásicas para el uso de albendazol como alternativa a la cirugía incluyen pacientes inoperables, casos con recurrencias post-quirúrgicas o presencia de quistes múltiples, demostrando superioridad frente a placebo, tanto en quistes hepáticos como pulmonares ${ }^{28,29}$. Sin embargo, en la práctica clínica es raro observar que los pacientes sean inoperables para justificar esta opción. Albendazol es importante en casos de cirugía conservadora hepática para disminuir la tasa de recurrencia ${ }^{15}$ y no existe una evidencia equivalente para quistes pulmonares que haya demostrado su eficacia por lo que su uso es convencional ${ }^{30}$. El rol de albendazol en cirugía hepática radical es desconocido ya que el riesgo de recurrencia es muy bajo y ello puede explicar porqué este compuesto no fue usado universalmente.

\section{Conclusiones}

Los resultados de este trabajo demuestran que aunque en forma esporádica, la hidatidosis es una enfermedad vigente en Chile y que afecta incluso a la población infantil. Sus formas habituales de presentación son la hepática y pulmonar, y en muchos casos es una enfermedad en múltiples sitios. Otros hallazgos importantes indican que los pacientes afectados no pertenecen al mundo rural aunque pueden tener una relación con éste, que los síntomas son inespecíficos en la fracción que los presenta aunque ocasionalmente pueden presentarse claves diagnósticas y que el inmunodiagnóstico tiene un rol de apoyo pero que no descarta la enfermedad en caso de una prueba negativa por la sensibilidad limitada de las técnicas actuales. El tratamiento de la hidatidosis sigue siendo esencialmente quirúrgico con la aplicación de técnicas que aseguran una baja tasa de recurrencias. Sin embargo, la tasa global de complicaciones, las re-hospitalizaciones por este tipo de causas y las estadías prolongadas no fueron infrecuentes en esta serie y subrayan la carga de enfermedad que tiene esta histoparasitosis. Además, sin complicaciones, la necesidad de re-hospitalización persiste para extirpar parte de los numerosos quistes hidatídicos que afectan a una parte de estos pacientes.
Agradecimientos: Los autores de este trabajo desean agradecer a la Sra. Marcela Muñoz y al Sr. Héctor Vergara de la Unidad de Bioestadística del Hospital Militar de Santiago por su gran apoyo para identificar los pacientes que sirvieron para preparar este manuscrito.

\section{Resumen}

Introducción: La hidatidosis es una enfermedad endémica en Chile. Se presenta la experiencia clínica de un hospital en Santiago, desde 1996 al 2010. Métodos: Los casos fueron identificados usando las bases de datos institucionales y se analizaron las características clínicas y patológicas, el tratamiento y la evolución en forma retrospectiva. Resultados: Se identificaron 23 pacientes intervenidos en 30 oportunidades. La mediana de edad fue de 40 años (rango 5 a 73) y 76,5\% visitó o residía en zonas endémicas en Chile. Los quistes abdominales fueron predominantes $(78,3 \%)$ y la mayor parte de los pacientes eran sintomáticos (73,9\%). Un 42,9\% de aquellos con quistes sintomáticos torácicos reportó eliminación de vesículas con la tos. Un 41,2\% presentó eosinofilia y $57,1 \%$ tuvo serología positiva para hidatidosis. Todos los pacientes fueron tratados quirúrgicamente, en $60,9 \%$ en combinación con albendazol. La mayoría de los quistes hepáticos fueron tratados con cirugía radical $(88,9 \%)$ y los pulmonares predominantemente con quistostomía y capitonaje (83,3\%). Un $24 \%$ de los quistes eran fértiles. La morbilidad post-quirúrgica fue frecuente $(37,9 \%)$ y $13,3 \%$ de la serie requirió reingresos por esta causa. Cerca de $25 \%$ de los pacientes estuvo hospitalizado por más de 14 días, un fenómeno asociado con la presencia de fiebre por complicación séptica al ingreso $(p<0,05)$. No se registraron recurrencias en la fracción de pacientes seguidos por más de dos años. Conclusiones: La hidatidosis afecta principalmente a adultos en su cavidad abdominal. Aunque los pacientes fueron tratados exitosamente con cirugía, las complicaciones, estadías prolongadas y reingresos por esta causa no fueron infrecuentes.

\section{Referencias}

1.- $\quad$ Eckert J, Gemmell M A, Meslin F-X, Pawlowski Z S. WHO/OIE Manual on echinococcosis in humans and animals: a public health problem of global concern. 2001. Disponible en: whqlibdoc.who.int/ publications/2001/929044522X.pdf

2.- Galdámez O, Cortés P, Vargas D, Rodríguez J, Vega F, Pérez C, et al. Variables epidemiológicas asociadas a la hidatidosis en población rural asintomática. Parasitol Día 1997; 21: 7-13.

3.- Moro P, Schantz P M. Cystic echinococcosis in the Americas. Parasitol Int 2006; 55 (Suppl): S181-6.

4.- Serra I, Araya C, Araneda J. Evolución epidemiológica de la hidatidosis humana en Chile, 1965-1988. Impacto de programas ganaderos en las regiones XI y XII. Rev Med Chile 1983; 121: 343-9.

5.- Godoy K, Galvez G, Tassara R. Frecuencia de hidatidosis en los principales centros quirúrgicos de Santiago de Chile, comparación con notificación al Ministerio de Salud (1988-1997). Bol Hosp San Juan de Dios 2000; 47: 309-13. 6.- Schenone H, Contreras M D C, Salinas P,
Sandoval L, Saavedra T, Rojas A. Hidatidosis humana en Chile. Seroprevalencia y estimación del número de personas infectadas. Bol Chil Parasitol 1999; 54: 70-3.

7.- Lorca M, Garrido M, Contreras M D C, Salinas P, Alvarez C, Ruiz M, et al. Seroprevalencia de hidatidosis humana en la Región de Coquimbo, Chile. Parasitol Latinoam 2006; 61: 111-6.

8.- Cortés S, Valle C. Hidatidosis humana: Generalidades y situación epidemiológica en Chile según egresos hospitalarios y notificación obligatoria entre los años 2001 y 2005. Rev Chil 
Infectol 2010; 27: 329-35.

9.- Oddó D, Tagle R, Oberpauer B. Hidatidosis humana en material de biopsia. Parasitol Día 1989; 13: 64-8.

10.- Morakote N, Thamprasert K, Lojanapiwat B, Muttarak M. Cistic echinococcosis in Thailand with a special note on detection by serology in one family. Southeast Asian J Trop Med Public Health 2007; 38: 796-8.

11.- Manterola C, Cuadra A, Muñoz S, Sanhueza A, Bustos L, Vial M, et al. In a diagnostic test study the validity of three serodiagnostic tests in patients with liver echinococcosis. J Clin Epidemiol 2005; 58: 401-5.

12.- Sapunar J, Rappaport J, Sapunar J, Cumsille F. Quiste hidatídico: características clínicas, factores pronósticos y resultados quirúrgicos. Parasitol Día 1989; 13: 52-63.

13.- Carmena D, Benito A, Eraso E. Avances en el inmunodiagnóstico de la hidatidosis humana. Enferm Infecc Microbiol Clin 2007; 25: 263-9.

14.- Mercado R, Atías A, Astorga B, Lorca M. Reacción en doble difusión en agar con detección del arco 5 en el diagnóstico de hidatidosis. Bol Of Sanit Panam 1998; 105: 15963.

15.- Arif S H, Shams-Ul-Bari, Wani N A, Zargar S A, Wani M A, Tabassum R, et al. Albendazol as an adjuvant to the standard surgical management of hydatid cyst liver. Int J Surg 2008; 6: 448-51.

16.- Manterola C, Barroso M, Oberg C, Molina E,
Vial M, Fernández O. Alternativas quirúrgicas en el tratamiento de la hidatidosis hepática. Bol Chil Parasitol 1999; 54:13-20.

17.- Yüksel O, Akyürek N, Şahin T, Salman B, Azili C, Bostanci H. Efficacy of radical surgery in preventing early local recurrence and cavity-related complications in hydatid liver disease. J Gastroenterol Surg 2008; 12: 483-9.

18.- Rodríguez R, Muñoz C. Hidatidosis abdominal. Análisis de 60 casos operados. Rev Méd Maule 2006; 24: 4-8.

19.- Nabi M S, Waseen T. Pulmonary hydatid disease: What is the optimal surgical strategy? Int J Surg 2010; 8: 612-6.

20.- Bagheri R, Ziaollah Haghi S, Amini M, Sadat Fattahi A, Noorshafiee S. Pulmonary hydatid cyst: analysis of 1024 cases. Gen Thorac Cardiovasc Surg 2011; 59: 105-9

21.- Manterola C, Vial M, Melo A, Oberg C, Fonseca F. Viability and fertility of human hepatic hydatid cysts. World J Surg 2006; 30 : 227-32.

22.- Horton R J. Chemotherapy of Echinococcus infection in man with albendazole. Trans R Soc Trop Med Hyg 1989; 83 (1): 97-102.

23.- Davis A, Dixon H, Pawlowski Z S. Multicentre clinical trials of benzimidazole-carbamates in human cystic echinococcosis (phase 2) Bull World Health Org 1989; 67 (5): 503-8.

24.- Franchi C, Di Vico B, Teggi A. Long-term evaluation of patients with hydatidosis treated with benzimidazole carbamates. Clin Infect Dis 1999; 29: 304-9.

25.- Morris D L, Dykes P W, Marriner S, Bogan J, Burrows F, Skeene-Smith H, et al. Albendazole-objective evidence of response in human hydatid disease. JAMA 1985; 253: 2053-7.

26.- Gil-Grande L A, Rodríguez-Caabeiro F, Prieto JG, Sánchez-Ruano JJ, Brasa C, Aguilar L, et al. Randomised controlled trial of efficacy of albendazole in intra-abdominal hydatid disease. Lancet 1993; 342 (8882): 1269-72.

27.- Teggi A, Lastilla M G, De Rosa F. Therapy of human hydatid disease with mebendazole and albendazole. Antimicrob Agents Chemother 1993; 37: 1679-84.

28.- Keshmiri M, Baharvahdat H, Fattahi S H, Davachi B, Dabiri R H, Baradaran H, et al. Albendazole versus placebo in the treatment of echinococcosis. Trans R Soc Trop Med Hyg 2001; 95: 190-4

29.- Keshmiri M, Baharvahdat H, Fattahi S H, Davachi B, Dabiri R H, Baradaran H, et al. A placebo controlled study of albendazole in the treatment of pulmonary echinococcosis. Eur Respir J 1999; 14: 503-7.

30.- Koul P A, Singh A A, Ahanger A G, Wahid A, Sofi B A. Optimal duration of preoperative anti-helminthic therapy for pulmonary hydatid surgery. ANZ J Surg 2010; 80: 354-7. 